\title{
OHVIRA Syndrome_-Diagnostic Dilemmas and Review of Literature
}

\author{
Ritu Sharma ${ }^{1}$, Pinky Mishra², Shikha Seth ${ }^{3}$, Neema Agarwal ${ }^{4}$
}

\begin{abstract}
Obstructed hemivagina, ipsilateral renal agenesis (OHVIRA) syndrome, as well as didelphic uterus, is a rare, obstructed müllerian anomaly, affecting the physical, reproductive, sexual, and mental health of the patient. The diagnosis is often delayed because of unawareness of the fact that even common gynecological complaints like vaginal discharge can be associated with this rare entity. The most common symptoms at presentation, however, include pain in the abdomen and menstrual complaints. Therefore, while evaluating them, the possibility of this rare entity should be kept in mind; hence, emphasizing the importance of local examination. A 3D ultrasound and a magnetic resonance imaging are helpful in confirming the diagnosis. In this article, we report case series of OHVIRA syndrome with extreme clinical symptoms at presentation. We reviewed the literature and discussed various classifications used for this syndrome, including the newer classification based on the type of obstruction. The minimally invasive "vaginoscopic no-touch" technique of surgery is an alternative option available for adolescents where preservation of hymen seems to be important. Keywords: Didelphic uterus, Herlyn-Werner-Wunderlich syndrome (HWWS), Ipsilateral renal agenesis, Obstructed hemivagina, Obstructed müllerian anomalies.

Journal of South Asian Federation of Obstetrics and Gynaecology (2020): 10.5005/jp-journals-10006-1847
\end{abstract}

\section{BACKGROUND}

Female reproductive function depends on the intact genital tract along with a functional hypothalamic-pituitary-ovarian axis. Aberration in any of the systems may significantly affect a woman's reproductive potential, thereby negatively affecting her quality of life. Müllerian anomalies are congenital developmental anomalies of the female reproductive tract with an overall incidence, including both major and minor müllerian anomalies, of $7-10 \%$ while after excluding the minor ones the incidence is $2-3 \%{ }^{1,2}$ The incidence of recurrent abortions reaches up to $16 \% .^{3}$ These anomalies are often associated with one or more extragenital anomalies as well; most common among them are the renal anomalies (17.3\%) with renal agenesis contributing to $64.6 \%$ of them. Among patients with didelphic uterus, $29.1 \%$ had associated renal anomalies with $23.6 \%$ diagnosed to have renal agenesis and obstructed hemivagina. ${ }^{4}$ Other extragenital anomalies include skeletal anomalies (12-19\%), congenital heart disease, and gastrointestinal malformation (12\%). ${ }^{5}$

Obstructed hemivagina, ipsilateral renal agenesis (OHVIRA) syndrome or Herlyn-Werner-Wunderlich syndrome (HWWS) is one such complex congenital developmental anomaly of the genitourinary tract characterized by didelphic uterus, obstructed hemivagina, and ipsilateral renal agenesis. It was first reported in $1922 .{ }^{6}$ The estimated incidence of obstructed müllerian agenesis is $0.1-3.8 \%$ in the general population, which may be underreported due to the associated diagnostic dilemma. ${ }^{7}$

Here, we present two cases of OHVIRA syndrome with two extreme clinical presentations-the most common acute presentation with cyclical abdominal pain in one while an extremely rare delayed presentation with chronic vaginal discharge in the other.

\section{Case Description}

\section{Case 1}

A 21-year-old nulligravid married woman presented with complaints of continuous foul-smelling discharge per vagina for 1 year. She ${ }^{1-3}$ Department of Obstetrics and Gynaecology, Government Institute
of Medical Sciences, Gautam Buddha Nagar, Uttar Pradesh, India
${ }^{4}$ Department of Radio-diagnosis, Government Institute of Medical
Sciences, Gautam Buddha Nagar, Uttar Pradesh, India

Corresponding Author: Pinky Mishra, Department of Obstetrics and Gynaecology, Government Institute of Medical Sciences, Gautam Buddha Nagar, Uttar Pradesh, India, Phone: +91 9871340798, e-mail: drpinki22@gmail.com

How to cite this article: Sharma R, Mishra P, Seth S, et al. OHVIRA Syndrome-Diagnostic Dilemmas and Review of Literature. J South Asian Feder Obst Gynae 2020;12(6):421-426.

Source of support: Nil

Conflict of interest: None

attained menarche at 14 years of age, continued with normal menstrual cycles, and had normal sexual life without any complaints. General and systemic examinations were unremarkable with a normal body mass index (BMI) of 22. Her secondary sexual characters were well developed. Speculum examination revealed copious foul-smelling mucopurulent discharge with an anterolateral bulge on the right side of the vagina. The cervix could not be visualized. On bimanual examination, soft, fluctuant, nontender longitudinal swelling of about $5 \times 3 \mathrm{~cm}$ was felt on the right anterolateral aspect of the vagina, starting just above the introitus and extending up to the fornix. The discharge was increased on pressing the swelling which raised the suspicion of a small fistula but none was visualized. The cervix felt high up and the uterus was anteverted, bulky, and broad at the fundus with a depression at the middle, firm, nontender, and mobile. Bilateral fornices were nontender. Culture of the mucopurulent discharge showed growth of Klebsiella pneumoniae. Ultrasonography raised the possibility of either bicornuate or septate uterus and revealed a hypoechoic collection posterior to the cervix with an absent right kidney. The patient was advised a magnetic resonance imaging (MRI) from an outside facility which

(c) Jaypee Brothers Medical Publishers. 2020 Open Access This article is distributed under the terms of the Creative Commons Attribution 4.0 International License (https://creativecommons.org/licenses/by-nc/4.0/), which permits unrestricted use, distribution, and non-commercial reproduction in any medium, provided you give appropriate credit to the original author(s) and the source, provide a link to the Creative Commons license, and indicate if changes were made. The Creative Commons Public Domain Dedication waiver (http://creativecommons.org/publicdomain/zero/1.0/) applies to the data made available in this article, unless otherwise stated. 

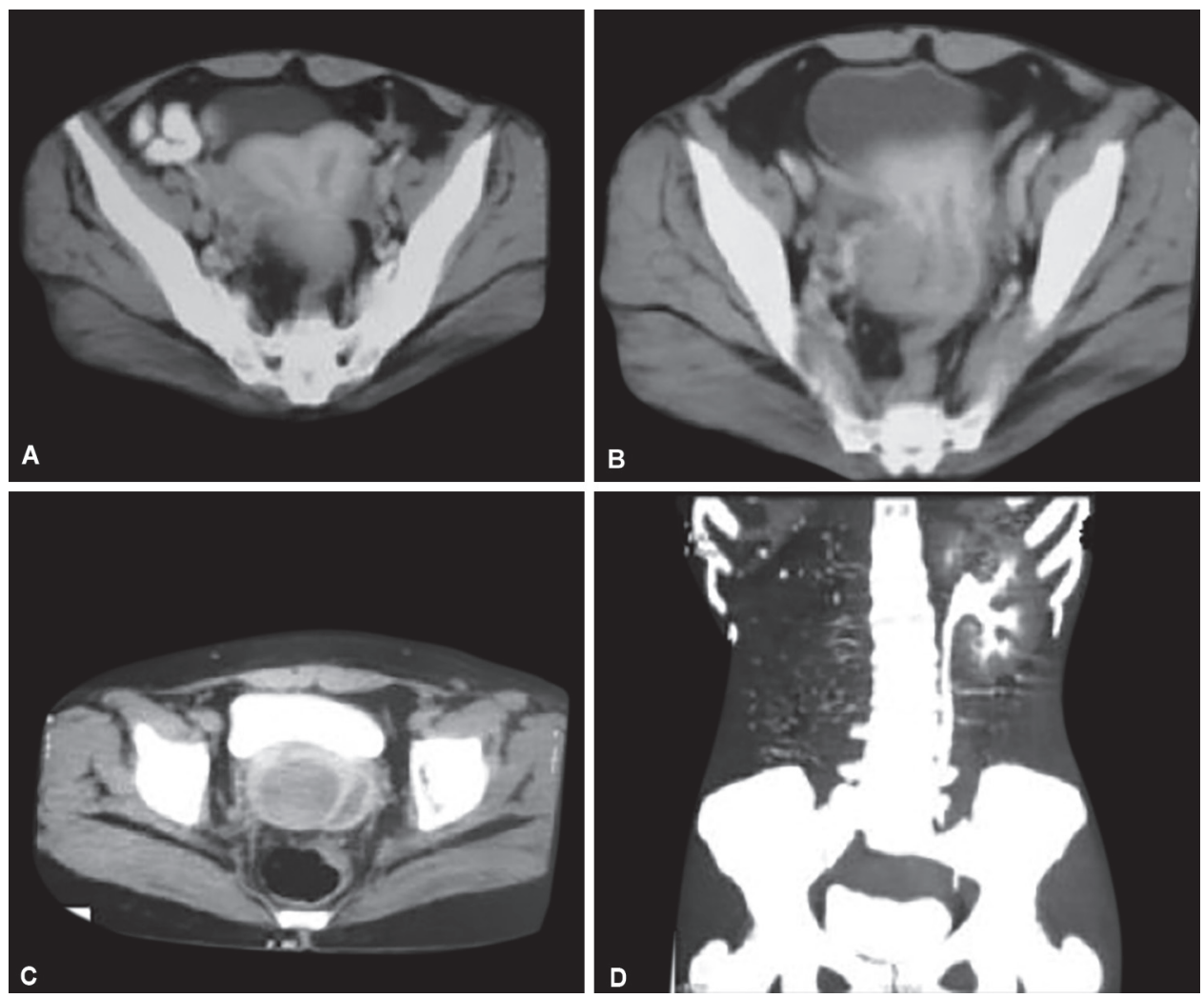

Figs 1A to D: (A) and (B) Contrast-enhanced axial CT image of pelvis shows two separate uterine cavities suggestive of didelphic uterus (C) Inferior section of the pelvis showing two vaginal cavities separated by a longitudinal septum with distended right hemivagina suggestive of right hematocolpos (D)-Coronal reconstructed image of CT abdomen with absent right kidney

she refused and got a contrast-enhanced computed tomography scan of the pelvis and lower abdomen which was readily available at our institute. The computed tomography (CT) scan findings revealed two well-defined endometrial cavities along with two hemivaginae, a hypodense collection of $35 \times 40 \mathrm{~mm}$ present in the right hemivagina with distal obstruction and absent right-sided kidney with compensatory hypertrophy on the left side (Fig. 1).

In view of the above findings, diagnosis of uterus didelphys with obstructed right hemivagina with ipsilateral renal agenesis, i.e., OHVIRA syndrome was made, and as per the American Society of Reproductive Medicine (ASRM) she was placed under class III, ${ }^{8}$ as per the European Society of Human Reproduction and Embryology (ESHRE) classification under class U3b C2 V2 ${ }^{9}$ (complete bicorporeal uterus, double "normal" cervix, longitudinal obstructing vaginal septum) and based on the type of obstruction-whether complete or partial, under class $2.1^{10}$ (partial resorption of the vaginal septum and communication between two vaginae).

The patient was counseled and underwent a vaginal septal resection with concomitant laparoscopy which revealed no evidence of adhesions, pelvic inflammatory disease (PID), or endometriosis. Postoperatively, there were no complications. She conceived twice within 1 year of surgery; the first pregnancy was a missed abortion and managed medically; the second one was successful with a full-term delivery via cesarean section.

\section{Case 2}

A 14-year-old girl, who attained menarche 1 year back, presented with cyclical abdominal pain and dysmenorrhea for 5 months. Her menstrual cycles were regular. On examination, the general condition was fair, BMI was 21.5, and secondary sexual characters were developed as per the age. On abdominal examination, a tender abdominal lump was palpable just above the pubic symphysis and the external genitalia were normal. Ultrasound showed a large collection behind the bladder with an unclear uterine morphology. MRI findings revealed uterus didelphys, a right-sided hemivaginal obstruction with blood collection, and an absent right-sided kidney (Fig. 2).

Based on the clinical and the imaging findings, a diagnosis of OHVIRA syndrome was made and as per ASRM she was placed under class III, ${ }^{8}$ as per the ESHRE classification under class U3b C2 V2, ${ }^{9}$ and based on the type of obstruction under class $1.1^{10}$ (blind hemivagina).

Parents were counseled and the patient was planned for an examination under anesthesia (EUA) followed by definitive treatment in the same sitting. EUA confirmed the findings and the vaginal septum was resected. There were no complications in the postoperative period and on follow-up.

\section{Discussion}

Müllerian duct anomalies are the developmental anomalies, resulting from defective development, defective fusion, or defective regression of the septum. Diagnosis of complex malformations is usually delayed, as due to their rarity they are not thought of or considered the cause of women's persistent or recurrent symptoms. OHVIRA syndrome is the result of an embryological arrest of the müllerian and mesonephric ducts at 8 weeks of gestation. The exact etiology of such malformation is unknown; however, genetic, environmental, and endocrine factors may have a role to play. 

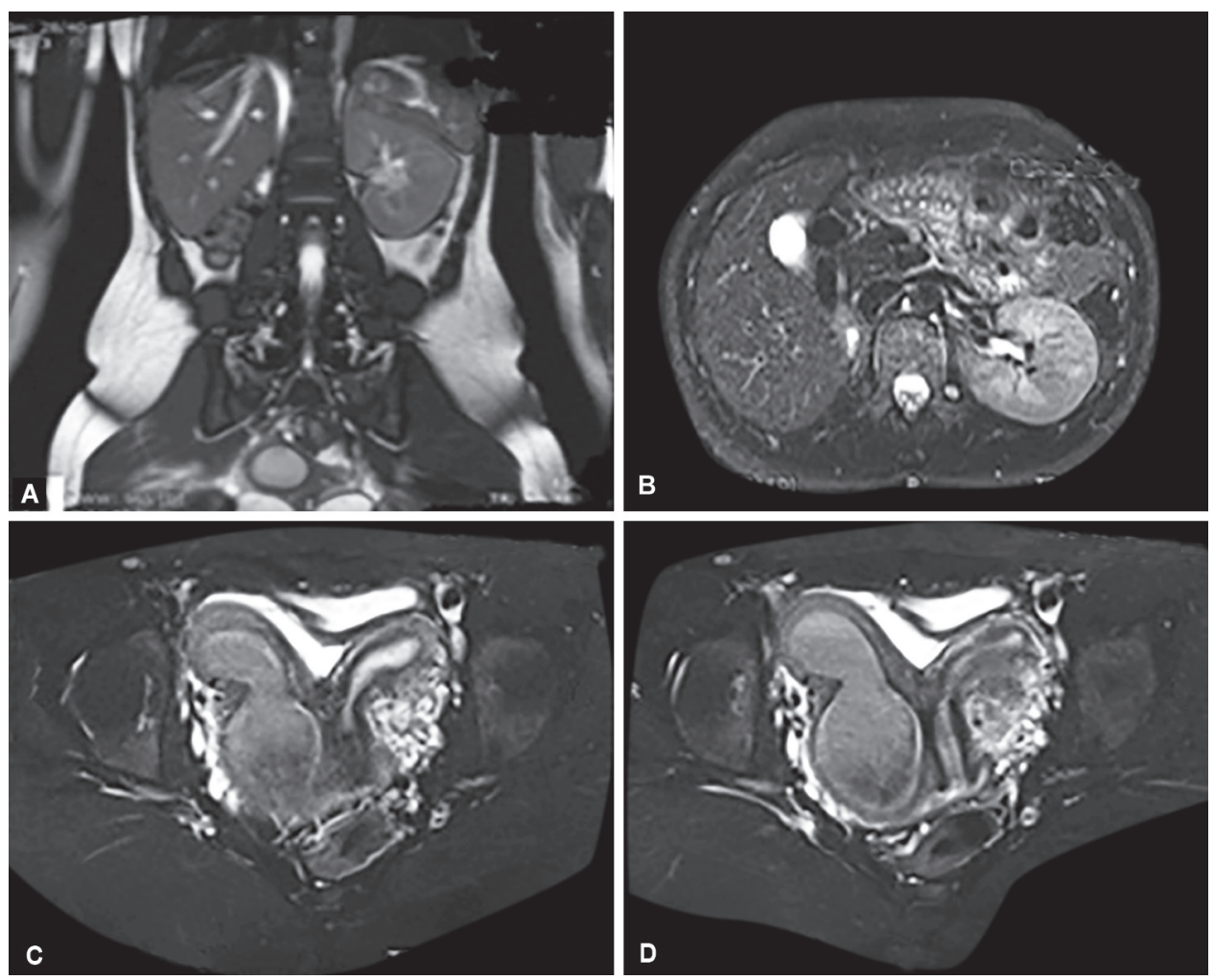

Figs 2A to D: Coronal MR image (A) and axial image (B) showing absent right kidney (C) and (D)-axial view showing two distinct uterine cavities; rightsided uterine cavity and right hemivagina are distended with mixed intensity fluid likely to be blood component suggestive of right hematocolpometra

Wingless integrated (Wnt) genes have been implicated in müllerian duct development but its role in this syndrome is unknown. ${ }^{11}$

The classical theory of development of female reproductive organs explains the development of the uterus, fallopian tubes, and the upper one-third of the vagina from the müllerian/ paramesonephric duct, and the lower two-thirds of the vagina from the urogenital sinus. According to this theory, however, the complex uterovaginal malformations associated with renal malformation are not explained completely. ${ }^{12}$

In 1992, Pedro Acien proposed a new embryological hypothesis that the vagina is an organ embryologically derived from the mesonephric or Wolffian ducts, mainly with the müllerian tubercle contributing to the vaginal epithelium only. The mesonephric ducts play an inducing role in the development of paramesonephric ducts (müllerian ducts), which fuse to form the uterus including the external cervical os. The caudal portion of the mesonephric ducts enlarges to form the sinovaginal bulbs at the level of cervical os which later fuse to form the vaginal plate. From the mesonephric duct opening in the urogenital sinus, the ureteric bud arises. So, in case of distal agenesis of a mesonephric duct, there will be agenesis of the ureteral bud on that side, hence ipsilateral renal agenesis, and due to its inducing function there will be associated uterine malformations. ${ }^{13-15}$ A congenital solitary kidney may undergo compensatory hypertrophy without any functional loss. ${ }^{16}$

OHVIRA syndrome in the literature has shown varied predilection related to the sides involved-some claiming rightsided abnormality being more common, some left sided, while others claimed equal prevalence on both sides. ${ }^{17-19}$

Taking into consideration the different classification systems of müllerian anomalies, OHVIRA syndrome is classified under class III as per the ASRM classification ${ }^{8}$ and under class U3b C2 V2 as per the ESHRE classification. ${ }^{9}$ At times, obstructed hemivagina and ipsilateral renal agenesis may be associated with a bicornuate or septate uterus (class U2b as per ESHRE/class IVa or Va as per ASRM); constituting OHVIRA variants. Based on a retrospective study including 70 patients by Zhu et al., OHVIRA syndrome is classified into two classes depending on whether the obstruction of the hemivagina is complete or incomplete (Flowchart 1). ${ }^{10}$

In a completely obstructed hemivagina, i.e., class 1, with the onset of menarche, there is a progressive collection of blood in the vagina, uterus, and fallopian tubes. If the obstruction is still not relieved, reflux of menstrual blood in the peritoneal cavity causes endometriosis, pelvic adhesions, and pelvic infections, leading to infertility. In patients with class 1, HWWS presents early after menarche with abdominal pain, abdominal lump, fever, peritonitis, and urinary tract obstruction. In patients with incomplete obstruction, i.e., class 2, it usually presents late with recurrent and persistent purulent vaginal discharge or PID. Diagnosis is delayed due to the occurrence of normal menstrual flow from the patent hemivagina. Sometimes, muco or hydrocolpos has been reported in infants ${ }^{20}$ and rarely vaginal clear cell carcinoma and cervical adenocarcinoma of the obstructed side have been reported. ${ }^{21}$

Diagnosis of OHVIRA syndrome requires a multimodal approach, which includes a detailed history, meticulous examination, and appropriate imaging studies. American Academy of Pediatrics recommends routine examination of the genitalia and EUA (if needed) in adolescents in case of genitourinary symptoms. ${ }^{22,23}$ Ultrasound and MRI are the established imaging modalities for diagnosing this condition with the ultrasound being the first-line option. A 3D ultrasound has $93 \%$ sensitivity and $100 \%$ specificity 
Flowchart 1: OHVIRA syndrome classification, clinical features and management ${ }^{10}$ (USG, ultrasonography; MRI, magnetic resonance imaging; EUA, examination under anesthesia; HSG, hysterosalpingograph)

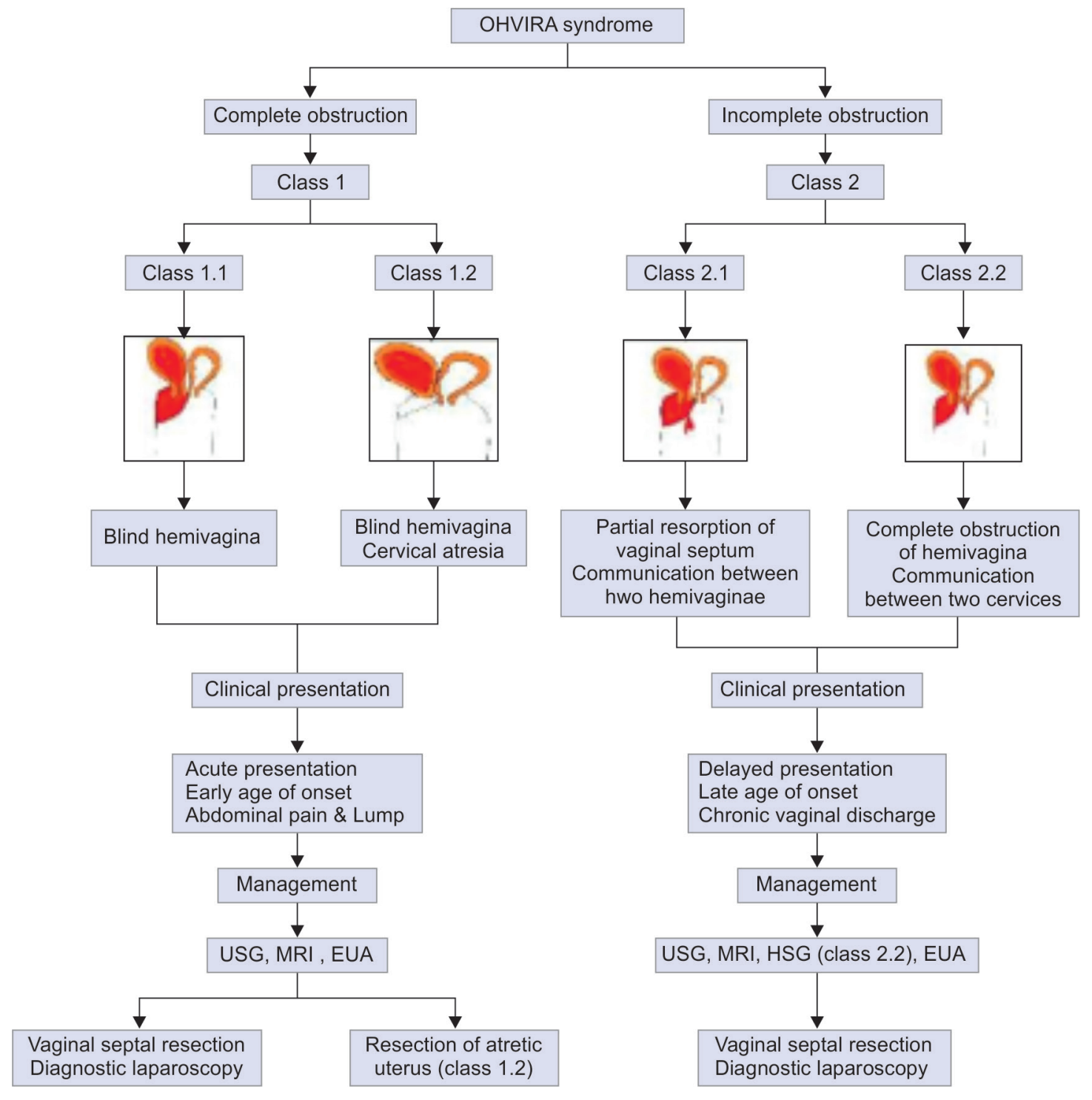

in the assessment of müllerian duct anomalies, hence a good alternative to MRI. However, expertise is required to diagnose the condition as the problem arises due to the small size of the uterus, nonreactive endometrium, and distended vagina in prepubertal and pubertal girls. ${ }^{24} \mathrm{MRI}$ is the gold standard investigation available to confirm the diagnosis, providing detailed information of both internal and external uterine anatomy, and to diagnose associated extragenital anomalies. It has $100 \%$ accuracy in detecting müllerian duct anomalies. ${ }^{25}$

Uterus didelphys accounts for $5 \%$ of all cases of müllerian anomalies and once diagnosed by imaging, it should be a dictum to look for the vaginal septum, as $75 \%$ of them are associated with a longitudinal vaginal septum. ${ }^{26}$ Also, it is advisable that whenever fetal renal anomalies are diagnosed on the antenatal scan look for obstructed müllerian anomalies in order to detect and treat them at the earliest. ${ }^{20}$ Hysterosalpingogram was used in the past quite often but now its role is limited to class 2.2 only to confirm communication between the two cervices. Laparoscopy can help in confirming the diagnosis, detection, and treatment of the associated complications and was considered to be the gold standard in the management of OHVIRA syndrome but due to the availability of better imaging facilities-3D ultrasound and MRI, it is no longer necessary in every case.

Obstructed anomalies, if not treated timely and adequately, may lead to chronic complications and negative psychological impact, thereby impairing a woman's quality of life. Awareness, familiarity, and a high index of suspicion would aid in early diagnosis and timely relief of obstruction with preservation of reproductive potential. Though OHVIRA syndrome is a complex and rare anomaly, its treatment, however, is relatively simple, comprising either resection or excision of the vaginal septum. Only in class 1.2 (cervicovaginal atresia with complete obstruction) would ipsilateral hysterectomy, either abdominal or laparoscopic, be required as in these cases apart from an obstructing vaginal septum, there is obstruction at another higher level, i.e., cervix. ${ }^{10}$ For those who refuse partial hysterectomy, the option of uterovaginal canalization but adequate counseling regarding restenosis of the neocervix and future complication is must. ${ }^{27}$ 
Conventionally, surgical resection of the vaginal septum is done under direct vision. Catheterizing the bladder before starting the procedure helps define the anatomy and avoid accidental injury to the urethra and bladder. Aspiration of accumulated contents in the obstructed hemivagina aids in placing the initial incision. The extent of the obstruction can be demarcated by palpation and the whole septum is resected. The use of electrocautery is convenient and reduces blood loss. Vicryl 2-0 can be used for suturing. Laser is also an option. This conventional approach may cause hymenal rupture and sometimes vaginal tears in adolescents, which leads to postoperative pain and adhesion formation. To overcome these issues, minimally invasive "vaginoscopic no-touch technique" with excellent visualization has been introduced for a longitudinal vaginal septum resection. Using a hysteroscopic L-hook electrode, the vaginal septum is incised followed by placement of a 14/16 Fr Foley's catheter, inflated with $50-80 \mathrm{~mL}$ saline in the previously obstructed hemivagina. This technique entails a good surgical outcome. ${ }^{28}$

Because of the associated risk of ascending infection, simple incision and drainage of the obstructed hemivagina are not recommended. Medical management with continuous oral contraceptive pills or medroxyprogesterone can be advised to suppress menstruation until definitive surgery is planned. ${ }^{29,30}$ Postoperatively, antibiotics, analgesics, and progestin tablets for menstrual suppression are prescribed. Those who are at high risk for stricture or adhesion formation need bed rest, vaginal molds, indwelling catheter, and deep vein thrombosis prophylaxis. Psychological counseling with respect to the diagnosis, classification of anomaly, consequences, management options, and timing of the surgery is important and should not be underestimated because of the association of this syndrome with the sexual, reproductive, and social stigma. ${ }^{29}$

Differential diagnosis of other causes of outflow tract obstruction should be kept in mind, which includes imperforate hymen, transverse vaginal septum, longitudinal vaginal septum, and cervical atresia. Except imperforate hymen, surgical management in others is complex, demanding expertise.

Follow-up is essential in view of associated risk of adhesions, stenosis, and endometriosis. The patient may complain of mucus discharge as the vaginal epithelium on the side of obstruction is columnar and has not undergone squamous metaplasia, requiring appropriate counseling. Cervical screening by Papanicolaou's smear is recommended separately for each cervix.

In timely and adequately treated patients with OHVIRA syndrome, reproductive performance is consistent with that of a didelphic uterus. Pregnancy rate in obstructed müllerian anomalies varies from $37-40 \% .{ }^{31}$ In a study of 49 women with didelphic uterus and longitudinal vaginal septum, pregnancy has been reported in up to $94 \%$ of cases with $21 \%$ resulting in abortion; $24 \%$ preterm labor; cesarean section rate, however, was $84 \%$. In this series, $16.3 \%$ of patients were having OHVIRA syndrome. Hence, early diagnosis and treatment entail a good reproductive outcome of this rare syndrome. ${ }^{32}$ Patients with OHVIRA syndrome are reported to have hypertensive disorders in pregnancy twice as compared to those with isolated uterine anomalies. ${ }^{33}$

\section{CONCLUSION}

Vaginal discharge, one of the most common gynecological complaints, if persistent and resistant to treatment, can be the manifestation of a rare congenital anomaly-OHVIRA, which should always be ruled out as if left untreated there are serious consequences on the reproductive and mental health. Timely surgical intervention and psychological counseling are of utmost importance. There is a need to sensitize the gynecologist, pediatrician, and radiologist to this rare entity.

\section{Clinical Significance}

Timely diagnosis and treatment of this rare anomaly will help in improving the reproductive outcome and quality of life of the patient.

\section{References}

1. Acien P. Incidence of Mullerian defects in fertile and infertile women. Hum Reprod 1997;12(7):1372-1376. DOI: 10.1093/oxfordjournals. humrep.a019588.

2. Troiano RN, McCarthy SM. Mullerian duct anomalies: imaging and clinical issues. Radiology 2004;233:19-34. DOI: 10.1148/ radiol.2331020777.

3. Simon C, Martinez L, Pardo F, et al. Mullerian defects in women with normal reproductive outcome. Fertil Steril 1991;56(6):1192-1193. DOI: 10.1016/s0015-0282(16)54741-4.

4. Heinonen PK. Renal tract malformations associated with Müllerian duct anomalies. Clin Obstet Gynecol Reprod Med 2018;4(1):1-5. DOI: 10.15761/COGRM.1000207.

5. Pinsonneault O, Goldstein DP. Obstructing malformations of the uterus and vagina. Fertil Steril 1985;(44)2:241-247. DOI: 10.1016/ S0015-0282(16)48745-5.

6. Purslow CE. A case of unilateral haematocolpos, haematomethra and haematosalpinx. J Obstet Gynaecol Br Emp 1922;29(4):643. DOI: 10.1111/j.1471-0528.1922.tb16100.x.

7. Gidwani G, Falcone T. Congenital malformations of the female genital tract. Diagnosis and management. Philadelphia: Lippincott Williams \& Wilkins; 1999. p. 145-168.

8. Birmingham, Alabama. The American Fertility Society classifications of adnexal adhesions, distal tubal occlusion, tubal occlusion secondary to tubal ligation, tubal pregnancies, Mullerian anomalies and intrauterine adhesions. Fertil Steril 1988;49(6):944-955. DOI: 10.1016/s0015-0282(16)59942-7.

9. Grimbizis GF, Gordts S, Di SpiezioSardo A, et al. The ESHRE/ESGE consensus on the classification of female genital tract congenital anomalies. Hum Reprod 2013;28(8):2032-2044.DOI: 10.1093/humrep/ det098. PMID: 23771171; PMCID: PMC3712660.

10. Zhu L, Chen N, Tong JL, et al. New classification of Herlyn-WernerWunderlich syndrome. Chin Med J (Engl) 2015;128:222-225. DOI: 10.4103/0366-6999.149208.

11. Mullen RD, Behringer RR. Molecular genetics of Müllerian duct formation, regression and differentiation. Sex Dev 2014;8(5):281-296. DOI: 10.1159/000364935.

12. Moore K, Persaud T. In the developing human: clinically oriented embryology. In: The Urogenital System. 8th ed. Philadelphia: Springer; 2008. p. 243-283.

13. Acien P. Embryological observations on the female genital tract. Hum Reprod 1992;7(4):437-445.DOI: 10.1093/oxfordjournals.humrep.a137666.

14. Acien P. Reproductive performance of women with uterine malformations. Hum Reprod 1993;8(1):122-126. DOI: 10.1093/ oxfordjournals.humrep.a137860.

15. Acien P, Acien M, Ferrer MS. Complex malformations of the female genital tract. New types and revision of classification. Human Reprod 2004;19(10):2377-2384. DOI: 10.1093/humrep/deh423.

16. Kerecuk L, Schreuder MF, Woolf AS. Renal tract malformations: perspectives for nephrologists. Nat Clin Pract Nephrol 2008;4:312325. DOI: $10.1038 /$ ncpneph0807.

17. Vercellini $P$, Daquati $R$, Somigliana $E$, et al. Asymmetric lateral distribution of obstructed hemivagina and renal agenesis in women with uterus didelphys: institutional case series and a systematic literature review. Fertil Steril 2007;87:719-724. DOI: 10.1016/j. fertnstert.2007.01.173. 
18. Robson WLM, Leung AKC, Rogers RC. Unilateral renal agenesis. Adv Pediatr 1995;42:575-592.

19. Haddad B, Barranger E, Paniel BJ. Blind hemivagina: long-term follow-up and reproductive performance in 42 cases. Hum Reprod 1999;14(8):1962-1964. DOI: 10.1093/humrep/14.8.1962.

20. David A, Gudi SN, Shankar R. Herlyn-Werner-Wunderlich syndrome: premenarche. J South Asian Feder Obst Gynae 2017;9(2):207-210. DOI: 10.5005/jp-journals-10006-1496.

21. Watanabe Y, Etoh T, Nakai H. Adenocarcinoma of the lower female genital tract in patients with Herlyn-Werner-Wunderlich syndrome. AJOG 2012;e5-e6. DOI: 10.1016/j.ajog.2012.09.009.

22. Braverman P, Breech L. Committee on adolescence. Clinical reportgynecologic examination for adolescents in the pediatric office setting. Pediatrics 2010;126(3):583-590. DOI: 10.1542/peds.20101564.

23. de Groot JA, Tierney Wolgemuth BS, Sanfilippo J. Mullerian anomalies in the pediatric and adolescent population: diagnosis, counseling and treatment options. Ann Infert Rep Endocrin 2019;2(1):1016. Available at: http://www.remedypublications.com/open-access/ mullerian-anomalies-in-the-pediatric-and-adolescent-populationdiagnosis-counseling-5171.pdf.

24. Kupesic S, Kurjak A. Ultrasound and Doppler assessment of uterine anomalies. In: Kupesic S, de Ziegler D, eds. Ultrasound and infertility. Pearl River, NY: Parthenon; 2000. p. 147-153.

25. Carrington $B M, H r i c a k H$, Nuruddin RN, et al. Mullerian duct anomalies: MR imaging evaluation. Radiology 1990;176:715-720. DOI: 10.1148/ radiology.176.3.2202012.
26. Olpin JD, Heilbrun M. Imaging of Mullerian duct anomalies. Clin Obstet Gynecol 2009;52(1):40-56. DOI: 10.1097/GRF.0b013e3181958439.

27. Mishra VV, Agarwal R, Bandwal P, et al. Management of hematometra in single horn of didelphic uterus with obstructed hemivagina and ipsilateral renal agenesis syndrome: a case report with brief review of literature. J South Asian Feder Obst Gynae 2016;8(3):231-235. DOI: 10.5005/jp-journals-10006-1423.

28. Cheng C, Subedi J, Zhang A, et al. Vaginoscopic incision of oblique vaginal septum in adolescents with OHVIRA syndrome. Sci Rep 2019;9:20042. DOI: 10.1038/s41598-019-56471-2.

29. Management of acute obstructive uterovaginal anomalies: ACOG Committee Opinion, Number 779. Obstet Gynecol 2019;133(6):12901291 DOI: $10.1097 /$ AOG.0000000000003282.

30. Committee on Adolescent Health Care. ACOG Committee Opinion No. 728:Müllerian agenesis: diagnosis, management, and treatment. Obstet Gynecol 2018;131(1):e35-e42. DOI: 10.1097/AOG.0000000000002458. PMID: 29266078.

31. Raga F, Bauset C, Remohi J, et al. Reproductive impact of congenital Müllerian anomalies. Hum Reprod 1997;10:2277-2281. DOI: 10.1093/ humrep/12.10.2277.

32. Heinonen PK. Clinical implications of the didelphic uterus: longterm follow-up of 49 cases. Eur J Obstet Gynecol Reprod Biol 2000;91(2):183-190. DOI: 10.1016/s0301-2115(99)00259-6.

33. Heinonen PK (2004) Gestational hypertension and preeclampsia associated with unilateral renal agenesis in women with uterine malformations. Eur J Obstet Gynecol Reprod Biol 114(1):39-43. DOI: 10.1016/j.ejogrb.2003.10.030. 